\title{
Correspondence: Some general points regarding Ledberg and Wennberg, BMC Medical Research Methodology 2014 April 27;14:58
}

\author{
Dankmar Böhning $^{1 *}$ and Peter G.M. van der Heijden ${ }^{2,3}$
}

\begin{abstract}
The purpose of this note is to contribute some general points on a recent paper by Ledberg and Wennberg (BMC Med Res Meth 14:58, 2014) which need to be rectified. They advocate the capture-removal estimator. First, we will discuss drawbacks of this estimator in comparison to the Lincoln-Petersen estimator. Second, we show that their evaluation of the Chao estimator is flawed. We conclude that some statements in Ledberg and Wennberg with respect to Chao's estimator and removal estimation need to be taken with great caution.
\end{abstract}

\section{Main text}

In a recent paper, Ledberg and Wennberg [1] propose to use the capture-removal estimator (Otis et al. [2]; Seber [3]; Borchers et al. [4]; ch. 5) for estimating the size of a hidden population from register data. It is assumed that a register has registrations from $M$ occasions with $M>1$. These occasions refer to different points in time so that they are chronologically ordered. The approach, at any occasion, consists of considering only new registrations and ignore those that have been identified before. Under the assumption that registration is independent at occasions and probability of registration is homogeneous a likelihood function can be determined and maximized in the two parameters involved, the probability of registration and the size $N$ of the population. Below we will first show, for two occasions, that the capture-removal estimator can have drawbacks in comparison with the Lincoln-Petersen estimator. Then we will show that the evaluation of the Chao estimator, given by Ledberg and Wennberg, is flawed.

\section{$M=2$ occasions}

We consider the case of two occasions, $M=2$. This is the simplest possible case and also allows an easy

\footnotetext{
*Correspondence: d.a.bohning@soton.ac.uk

${ }^{1}$ Southampton Statistical Sciences Research Institute, Mathematics and Medical Statistics, University of Southampton, Southampton SO17 1BJ, UK Full list of author information is available at the end of the article
}

comparison with the Lincoln-Petersen estimator and the bias-corrected Chapman estimator (Borchers et al. [4]). Let, as in Ledberg and Wennberg [1], denote with $n_{1}$ all registrations at occasion 1 (here every occasion is a new registration) and with $n_{2}$ all registrations at occasion 2 that were not yet registered at occasion 1 . For the setting of $M=2$ occasions it is possible to derive the maximum likelihood estimate of $N$ in a closed form expression: $\hat{N}_{R}=\frac{n_{1}^{2}}{n_{1}-n_{2}}$, assuming that $n_{1}>n_{2}$ which may or may not be met in practice. We denote this estimator as $\hat{N}_{R}$, index $R$ for removal. For comparison, we consider the Lincoln-Petersen estimator given as $\hat{N}_{L P}=\frac{n_{1}\left(m+n_{2}\right)}{m}$ and the Chapman estimator given as $\hat{N}_{C h}=\frac{\left(n_{1}+1\right)\left(m+n_{2}+1\right)}{(m+1)}$.

We will show that the latter two estimators are the better choice in the following two situations: first, when the assumption of constant and occasion-independent inclusion probabilities of the capture-removal estimator are met, the Lincoln-Petersen and the Chapman estimators are generally more efficient. Second, when the assumption of homogeneous inclusion probabilities that underlies the capture-removal estimator is not met, the captureremoval estimator is biased whereas the Lincoln-Peterson and the Chapman estimators are not. However, when there is behavioral response, i.e. after an inclusion the probability of the next inclusion increases, the LincolnPeterson and the Chapman estimators are biased downwards whereas the capture-removal estimator might be 
less biased depending on the constellation of marginal distributions and occasion dependency. We note that in the biological literature the first condition is known as $M_{0}$ for the inclusion probability being constant over time (under which the removal estimator is derived) and the second condition is known as $M_{t}$ for the inclusion probability varying with occasions (under which the Lincoln-Petersen and Chapman estimators are derived), whereas behavioral response is $M_{b}$.

To illustrate we have done the following simulation study. The population size is $N=1000$ and we looked at different scenarios of registration probability. Let $p_{1}$ be the registration probability at occasion 1 and $p_{2}$ at occasion 2 , registration is independent from occasion. Table 1 shows the mean and standard deviation of the Lincoln-Petersen, the Chapman and the removal estimator, respectively, for different settings. All simulation results are based upon 1000 replications. In setting 1 and 2 of Table 1 we look at equal registration probabilities, but the second setting has smaller ones. Both give reasonable mean results but the Lincoln-Petersen and Chapman estimators have the smaller standard deviation. Also, the variance of both estimators increase if the registration probability decreases. For setting 3 and 4 of Table 1, registration probability for occasion 2 is larger than for occasion 1 . Here, the captureremoval estimator overestimates, in particular for setting 4 where it becomes almost useless. On the contrary, for setting 5 and 6 of Table 1, where the registration probability for occasion 2 is lower than for occasion 1 , the capture-removal estimator underestimates, for setting 6 quite seriously. In all six settings, the Lincoln-Petersen and the Chapman estimators are giving unbiased estimates although the variance increases when the registration probabilities become small. For fairness, we also show simulation results for two settings where the removal estimator is doing better than the Lincoln-Petersen and Chapman estimators. This is in the case of behavioral response, i.e. once observed for the first time the probability for a second observation increases. In setting 7 at the first occasion is 0.50 , but at the second occasion it increases to $0.30 /(0.30+0.20)=0.60$ for those who have been already observed. In setting 8 this second conditional probability increases from 0.50 to $.375 /(.375+.125)=0.75$. In these two situations the removal estimator outperforms the Lincoln-Petersen and Chapman estimators. The last two settings 9 and 10 are two further examples of behavioral response and show that also the capture-removal estimator can be seriously biased. The reason is that the capture-removal estimator, at least in the way it is used by Ledberg and Wennberg, requires that the conditional probability for capture at occasion 2 given no capture at occasion 1 has to be identical to the unconditional probability of capture at occasion 1 (see Borchers et al. [4], p.76) which is not fulfilled in these last two settings.

\section{Multiple occasions}

We would like to make a second point considering $M$ occasions. In the case of homogeneity and independence the probability of observing exactly $y$ registrations for a unit is given by the binomial $P(Y=y)=\left(\begin{array}{c}M \\ y\end{array}\right) p^{y}(1-p)^{M-y}$, where $p$ is the probability of a registration at any, fixed occasion. Then, the Chao estimator (Chao [5]) of hidden units, the frequency of units with exactly zero registrations, is given as $\hat{f}_{0}=\frac{M-1}{M} \frac{f_{1}^{2}}{2 f_{2}}$, which is asymptotically

Table 1 Simulation results for registration system with two occasions. $p_{11}$ is the probability for capture at occasion 1 and occasion 2 , $p_{10}$ is the probability for capture at occasion 1 but not at occasion 2, and so forth. The marginal probabilities for capture at occasion 1 and 2 are $p_{1}=p_{11}+p_{10}$ and $p_{2}=p_{11}+p_{01}$, respectively. In settings 1 to 6 inclusion on occasion 1 is independent of inclusion on occasion 2. In settings 7 and 8, occasions become dependent (odds ratio larger than 1) but the conditional probability for capture at occasion 2 given no capture at occasion 1 is identical to the unconditional probability for capture at occasion 1, the capture-removal estimator works fine. In settings 9 and 10, those conditional and unconditional probabilities are different and the capture-removal estimator breaks down

\begin{tabular}{|c|c|c|c|c|c|c|c|c|c|c|c|c|}
\hline \multirow[b]{2}{*}{ Setting } & \multirow[b]{2}{*}{$p_{1}$} & \multirow[b]{2}{*}{$p_{2}$} & \multirow[b]{2}{*}{$p_{11}$} & \multirow[b]{2}{*}{$p_{10}$} & \multirow[b]{2}{*}{$p_{01}$} & \multirow[b]{2}{*}{$p_{00}$} & \multicolumn{2}{|c|}{ LP } & \multicolumn{2}{|c|}{ Chapman } & \multicolumn{2}{|c|}{ Removal } \\
\hline & & & & & & & $\bar{N}_{L P}$ & $\mathrm{SD}$ & $\bar{N}_{C h}$ & $\mathrm{SD}$ & $\bar{N}_{R}$ & SD \\
\hline 1 & 0.5 & 0.5 & 0.25 & 0.25 & 0.25 & 0.25 & 1001 & 31 & 1000 & 31 & 1007 & 59 \\
\hline 2 & 0.3 & 0.3 & 0.09 & 0.21 & 0.21 & 0.49 & 1006 & 77 & 1000 & 76 & 1064 & 262 \\
\hline 3 & 0.5 & 0.6 & 0.30 & 0.20 & 0.30 & 0.20 & 1002 & 27 & 1001 & 27 & 1271 & 113 \\
\hline 4 & 0.3 & 0.35 & 0.105 & 0.195 & 0.245 & 0.455 & 1004 & 66 & 1000 & 65 & 1825 & 6475 \\
\hline 5 & 0.5 & 0.3 & 0.15 & 0.35 & 0.15 & 0.35 & 1000 & 48 & 998 & 47 & 714 & 21 \\
\hline 6 & 0.3 & 0.1 & 0.03 & 0.27 & 0.07 & 0.63 & 1021 & 155 & 999 & 146 & 392 & 17 \\
\hline 7 & 0.5 & 0.55 & 0.30 & 0.20 & 0.25 & 0.25 & 955 & 28 & 955 & 28 & 1003 & 57 \\
\hline 8 & 0.5 & 0.625 & 0.375 & 0.125 & 0.25 & 0.25 & 834 & 18 & 834 & 18 & 1009 & 56 \\
\hline 9 & 0.3 & 0.1 & 0.065 & 0.235 & 0.035 & 0.665 & 464 & 34 & 462 & 34 & 340 & 15 \\
\hline 10 & 0.5 & 0.5 & 0.4 & 0.1 & 0.1 & 0.4 & 625 & 17 & 626 & 17 & 625 & 17 \\
\hline
\end{tabular}


unbiased where asymptotics refer to $N$ and $f_{x}$ is frequency of units with exactly $x$ registrations. If $M$ is large it becomes the Chao estimator under Poisson sampling that is mentioned in Ledberg and Wennberg [1]. Clearly, the asymptotic unbiasedness result holds under homogeneity for any $M$, although the variance is smaller the larger $M$ is. There is also a biased-corrected version of Chao's estimator which reduces the small-sample bias under homogeneity, but for the sake of brevity we will not consider this bias-corrected estimator here. If there is heterogeneity in registration (this is referred to in the biological literature as $\left.M_{h}\right)$ then the result in Chao $([5,6])$ says that the estimator represents only a lower bound. This is also why Chao's estimator is also called the lower bound estimator. The underestimation bias is mostly small in comparison with other estimators that assume homogeneity. When $M$ is becoming larger the underestimation bias will also become smaller in an absolute sense. This occurs since the number of registered users $s=\sum_{i=1}^{M} f_{i}$ will become larger and the number of units not observed becomes smaller. This is because the probability of a unit remaining undetected will decrease with $M$ becoming large as can be seen from the following equation (binomial sampling under heterogeneity) $P(Y=0)=\sum_{j=1}^{J}(1-$ $\left.p_{j}\right)^{M} w_{j} \rightarrow 0$, as $M$ approaches $\infty$, where $p_{j}$ is the probability of registration in subpopulation $j$ and $w_{j}$ its associated subpopulation weight. Hence the following statement in the discussion of Ledberg and Wennberg [1] is unsound and needs to be revised:

Assume that registrations are followed over a period of time. Since estimates obtained by Chao's estimator should not strongly depend on the duration of the time period used, similar estimates should be obtained if the first half of the time period is used compared to if the whole time period is used.

Clearly, in the case of heterogeneity, the bias of Chao's estimator is smaller when a larger number of occasions is considered. To illustrate this point we have done a small simulation experiment. The true $N$ is 200 and all results are based upon 1000 replications. In the first setting, the population is homogeneous with $p=0.1$. In the second, setting we assume a subpopulation structure with equal weights $w_{j}=0.5$ allocated to $p_{1}=0.3$ and $p_{2}=0.05$. In Table $2, \bar{s}$ denotes the mean of the number of observed different users (averaged over the 1000 replications), $\bar{f}_{0}$ denotes the mean of the estimated frequency of hidden units $f_{0}$ (averaged over the 1000 replications), and $\operatorname{SD}\left(\hat{f}_{0}\right)$ its estimated standard deviation. For an unbiased estimate we should have that $\bar{s}+\bar{f}_{0}=N$, in our case 200 . For setting 1 , this is practically the case, although the standard deviation is better for $M=10$ in comparison to $M=5$. For setting 2, the estimator experiences bias, with a value
Table 2 Chao's estimator for registration system with M occasions and true $N=200$

\begin{tabular}{|c|c|c|c|c|c|c|c|c|c|c|}
\hline \multirow[b]{2}{*}{ Setting } & \multirow[b]{2}{*}{$p_{1}$} & \multirow[b]{2}{*}{$p_{2}$} & \multicolumn{4}{|c|}{$M=5$} & \multicolumn{4}{|c|}{$M=10$} \\
\hline & & & $\bar{s}$ & $\bar{f}_{0}$ & $\bar{N}$ & $\mathrm{SD}\left(\hat{f}_{0}\right)$ & $\bar{s}$ & $\bar{f}_{0}$ & $\bar{N}$ & $\mathrm{SD}\left(\hat{f}_{0}\right)$ \\
\hline 1 & 0.1 & 0.1 & 82 & 120 & 202 & 52 & 130 & 70 & 200 & 20 \\
\hline 2 & 0.3 & 0.05 & 106 & 40 & 156 & 13 & 137 & 29 & 166 & 10 \\
\hline
\end{tabular}

of 54 for $M=5$ and a value of 34 for $M=10$, the latter being clearly smaller than the former. The reason for this bias is that the lower bound of Chao's estimator will only be reached under homogeneity and in setting 2 there is heterogeneity. In such a practical situation it pays off to have a longer observation period. However, if the observation period is taken to be too long, the violation of the assumption of a closed population may become more likely.

We conclude that the capture-removal estimation approach can be useful under certain (but not all) constellations of behavioral response. However, it is sensitive to violations of registration homogeneity and independence of occasions, as pointed out in Borchers et al. ([4]; Ch. 5). If the Lincoln-Petersen approach can be used instead it is the better choice for two reasons: it does not require identical registration probabilities in the occasions (the marginal distributions in Table 3 do not need to be equal), and secondly, makes full use of the available information in Table 3 (the removal estimator uses only the marginal information for occasion 1), so that the Lincoln-Petersen estimator has the better efficiency.

It has been seen that some statements in Ledberg and Wennberg on Chao's estimator, in particular on its independence of the number of occasions, need to be revised, especially, if there is population heterogeneity. Even if there is homogeneity the variation for the entire period will be considerably smaller than for the first half-period. It might be better to compare the Chao estimator for different periods of equal size.

From our perspective, Chao's estimator remains as one of the most useful estimators in the area. We recently proposed a generalization of Chao's estimator that can take covariates into account (Böhning et al. [7]). Thus observed population heterogeneity can be modelled and the lower

Table 3 Registration system with two occasions

\begin{tabular}{|c|c|c|c|c|}
\hline & \multicolumn{4}{|c|}{ Occasion 2} \\
\hline & & 1 & 0 & \\
\hline & 1 & $m$ & $n_{1}-m$ & $n_{1}$ \\
\hline \multicolumn{5}{|l|}{ Occasion 1} \\
\hline & 0 & $n_{2}$ & $x$ & \\
\hline & & $m+n_{2}$ & & $N$ \\
\hline
\end{tabular}


bound provided by this covariate adjusted estimator will be closer to the true population size than the unadjusted estimator.

\section{Response}

by Anders Ledberg and Peter Wennberg

Corresponding author: Anders Ledberg

Email: anders.ledberg@sorad.su.se

Address: Centre for Social Research on Alcohol and Drugs, SoRAD Stockholm University, SE-10691 Stockholm, Sweden

\section{Introduction}

We are happy about the attention our publication "Estimating the size of hidden populations from register data" [1] has received and would like to use this opportunity to clarify what our paper is about and what it is not about.

\section{What our paper is about}

In our paper we are considering the problem of estimating the size of an incompletely sampled population. The particular case we have in mind is that when a given individual in the population has constant probability, per unit time, of being first registered, but once registered the probability of future registrations might change, perhaps radically. (We use 'registered' in a general sense here; the analogous concept in the ecological literature would be 'captured', or 'trapped'). This case is of interest to us since we believe that it could serve as an approximate model for epidemiological data. As an example, consider the "population" of heavy drug users. Assume that there is a constant probability that heavy drug use leads to contact with the health care system for the first time (and a registration). One possible outcome of such a contact is that the client enters a treatment program that implies regular contacts with the health care system (for example methadone maintenance treatment). Consequently, the probability that this particular individual is registered again is very high (close to one). Indeed, that the probability of registration is history dependent seems to us a generic feature of this type of data. In the literature on population estimation in ecology this history dependence is often called behavioral response [e.g. [2]]. In keeping with this terminology (of [2]) we call this scenario Model $M_{b}$. In other words, our paper suggests modeling (some types of) epidemiological data using Model $M_{b}$, and to use the maximum likelihood estimator derived under this model [3].

In our paper we evaluate the performance of this maximum likelihood estimator under the scenario we consider, and show when it is applicable, and when it is not (Figure 2 in [1]). In particular, we show that for the estimator to be useful a certain fraction of the population should be sampled, and this fraction depends on the total size of the population (Figure 2 in [1]). An important result is that the estimator is robust under moderate heterogeneity with respect to the probabilities of first registration of different individuals, i.e. they need not be identical for the estimator to be useful (see Figure 3 in [1]). Another contribution is that we show that some other estimators, that have been used on data that could be reasonably modeled using Model $M_{b}$, can have a substantial bias when applied to data from Model $M_{b}$. In particular, we show that an estimator that can be derived assuming that the data follow a truncated Poisson distribution, can have a substantial bias, and that this bias can be positive, i.e. it might lead to an overestimation of the population size (see Figure 6 in $[1])$.

\section{What our paper is not about}

Estimating the size of hidden populations is a problem that has been treated by many authors and there are many different methods in use. The basic idea in deriving a measure (an estimator) is to start with a particular scenario (model) for the registrations, and from this model derive an estimator. Thus, key aspects of a real situation (e.g. drug users interacting with the health care system) are captured in an idealized model (Model $M_{b}$ in our case), and given this model an estimator is derived (maximum likelihood estimator in our case). The estimator is then strictly valid only under the model considered. We certainly do not suggest that the maximum likelihood estimator should be used if the data at hand are better described by other models (such as Models $M_{0}$ or $M_{t}$, for example). Indeed, that an estimator derived under model $A$ does not perform well when applied to data generated under model $B$ is neither surprising nor informative for its performance under model $A$.

Our paper does not provide an evaluation of other estimators, and our evaluation of the maximum likelihood estimator is done only under some particular scenarios. We have no particular attachment to the estimator we propose but for the type of data we are interested in it still seem a most reasonable choice (given, of course, that a sufficient fraction of the population is sampled). Böhning and van der Heijden do not suggest another estimator that works better in this case, something we interpret as them being in tacit agreement with us. Perhaps contrary to these workers, we do not believe in a "universal estimator" that should always be used. Rather, as we suggest in our paper, application of several estimators, relying on different assumptions, might provide complementary information about the data at hand and might help in getting more reliable estimates.

\section{Competing interests}

The authors declare that they have no competing interests. 


\section{Acknowledgements}

This research has been financed by the Swedish Council for Working Life and Social Research (FAS 2006-1523).

\section{References}

1. Ledberg A, Wennberg, P. Estimating the size of hidden populations from register data. BMC Med Res Methodol 2014;14(58):58.

2. Otis D, Burnham K, White G, Anderson D. StatisticalInference From Capture Data On Closed Animal Populations. Wildlife Monogr 1978;(62):7-135.

3. Moran P. A Mathematical Theory Of Animal Trapping. Biometrika 1951;38(3-4):307-311.

\section{Competing interests}

The authors declare that they have no competing interests.

\section{Authors' contributions}

DB carried out all analysis and provided the first draft of the paper. PvdH edited and finalized the manuscript. Both read and approved the final manuscript.

\section{Author details}

${ }^{1}$ Southampton Statistical Sciences Research Institute, Mathematics and Medical Statistics, University of Southampton, Southampton SO17 1BJ, UK.

${ }^{2}$ Southampton Statistical Sciences Research Institute, University of Southampton, Southampton SO17 1BJ, UK. ${ }^{3}$ Department of Methods and Statistics, Social Sciences, University of Utrecht, Utrecht, Netherlands.

Received: 16 August 2014 Accepted: 16 June 2015

Published online: 07 July 2015

\section{References}

1. Ledberg A, Wennberg P. Estimating the size of hidden populations from register data. BMC Med Res Methodol. 2014;14:58.

2. Otis DL, Burnham KP, White GC, Anderson DR. Statistical inference from capture data on closed animal populations. New York: Wiley; 1978.

3. Seber GAF. The estimation of animal abundance, 2nd Ed. London: Charles Griffin; 1982.

4. Borchers DL, Buckland ST, Zucchini W. Estimating animal abundance: closed populations. London: Springer; 2002.

5. Chao A. Estimating the population size for capture-recapture data with unequal catchability. Biometrics. 1987;43:783-91.

6. Chao A. Estimating population size for sparse data in capture-recapture experiments. Biometrics. 1989;45:427-38.

7. Böhning D, Lerdsuwansri R, Vidal-Diez A, Viwatwongkasem C, Arnold M. A generalization of Chao's estimator for covariate information. Biometrics. 2013;69:1033-42.

Submit your next manuscript to BioMed Central and take full advantage of:

- Convenient online submission

- Thorough peer review

- No space constraints or color figure charges

- Immediate publication on acceptance

- Inclusion in PubMed, CAS, Scopus and Google Scholar

- Research which is freely available for redistribution 